\title{
A nonlinear regression approach to estimating signal detection models for rating data
}

\author{
CHING-FAN SHEU \\ DePaul University, Chicago, Illinois \\ and \\ ANDREW HEATHCOTE \\ University of Newcastle, Newcastle, New South Wales, Australia
}

\begin{abstract}
This paper considers a regression approach to estimating signal detection parameters for rating data. The methodology is based on the statistical modeling of ordinal data and requires only standard statistical software such as SAS (SAS/STAT User's Guide, 1999) for computation. The approach is more efficient than the current practice of extracting the parameter estimates with the use of specializedsoftware and analyzing the estimates with the use of a standard statistical package. It greatly facilitates exploration of the effects of covariates on model parameters. The method is illustrated using a published data set from a single factor multiple-alternative perceptual task, and data from a more complex factorial design examining recognition memory rating data.
\end{abstract}

This paper presents a method for fitting covariateadjusted signal detection models to rating data from discrimination experiments. In the regression framework, treatment conditions can be coded as indicator covariate variables so that the effects of experimental factors on parameter estimates of the signal detection model can be assessed directly from the regression coefficients of a generalized (ordinal) regression equation.

The ability to directly analyze effects of covariates (i.e., factors in experimental designs) on parameters provides a flexible approach to data analysis using theory of signal detectability (MacMillan \& Creelman, 1991). For example, this approach allows simultaneous fitting of all conditions in a complex experiment rather than comparison of results from multiple hit versus false alarm ROC analyses. It also allows a nested (full versus reduced) model comparison to be conducted without having to rewrite the software for the estimating procedure when a different reduced (nonsaturated) model is considered.

A widely used approach to fitting the confidence ratings resulting from a discrimination task is to assume a (latent) normal model (Green \& Swets, 1974) and to estimate the parameters separately for each pair of ratings from signal and noise conditions using a maximum likelihood method (Dorfman \& Alf, 1969). Kijewski, Swensson, and Judy (1989) extended this approach to simultaneously fit rating data from more than two alternatives.

This work was supported in part by a paid-leave program from the University Research Council of DePaul University to C.-F. Sheu and partly by a research visitor grant from the University of Newcastle, New South Wales, Australia. Correspondence concerning this article should be addressed to C.-F. Sheu, Department of Psychology, DePaul University, 2219 North Kenmore Ave., Chicago, IL 60614-3522 (e-mail: csheu@depaul.edu).
Their fitting algorithm requires second-order derivative equations for the maximum-likelihood estimation. Their work illustrates the difficulties an experimenter faces in applying signal detection models for data collected from factorial experimental designs. The experimenter must be able to differentiate with respect to the unknown parameters in the maximum-likelihoodestimating equations and implement the calculations in a computer language. Such an approach is not practical since it requires the construction of a specialized procedure for each experimental design. The approach is also inefficient. The researcher typically wishes to assess the effect of a treatment condition on the parameter estimate, such as the difference between signal and noise means. The standard approach requires that this is accomplished in two stages. First, the parameter estimates are extracted from noise and signal conditions of an experiment with the use of some specialized computer programs, and then a standard statistical procedure such as analysis of variance is performed on the estimates as if they were raw data. A more efficient approach would allow the effects of factors in an experimental design on parameter estimates to be assessed directly.

Tosteson and Begg (1988) proposed a regression approach to estimate receiver-operating-characteristiccurves in radiology. Their ordinal regression methodology is built on the framework of the generalized linear models (McCullagh \& Nelder, 1989) and allows the estimation of location and scale parameters to be adjusted for relevant covariates. Tosteson and Begg used a specialized computer program for their analysis. DeCarlo (1998) discussed the connection between signal detection theory and generalized linear models and presented sample SAS and SPSS programs (see SAS/STAT User's Guide, 1999) for well-known data sets in signal detection literature. Un- 
fortunately, the often used unequal variance normal model is not of the generalized linear type, and there is no builtin procedure in general-purpose statistical software to estimate such model parameters automatically.

Some researchers have estimated the parameters of the unequal variance signal detection model with the use of multiple linear regression on transformed cumulative ratings proportions (e.g., Ratcliff, McKoon, \& Tindall, 1994). Which transformation is used depends on the underlying form of the latent distribution. This approach has several weaknesses. First, ratings from all but one condition are used as predictors and so are assumed to be measured without error, a clearly wrong assumption. Further, error is minimized by regression on the remaining condition, giving it undue influence. Finally, each data point is equally weighted, but clearly some have much greater estimation variance than others. For reasonably accurate responding, for example, there are usually few high confidence errors and so data points for these ratings are very variable. The problem is exacerbated by the strongly nonlinear effect of the transformation when cumulative probabilities are near 0 or 1 . As a consequence, this approach is likely not to be robust. However, none of these weaknesses of an ad-hoc linear regression approach applies to maximum likelihood estimation. It is well known that maximum likelihood estimation can be achieved by means of nonlinear least squares (Jennrich \& Moore, 1975). In this paper, we follow the method suggested by Cox (1984), who treated the problem of maximizing likelihood as a regression problem and used nonlinear regression programs that are available in standard statistical packages to estimate signal detection parameters.

We believe that the requirement of using specialized software prohibits the regression methodology from being widely applied by psychologistsfor the analysis of signal detection experiments. A goal of this paper is to bridge the gap between standard regression modeling of ordinal data and the practice of ROC data analysis in psychology. We acknowledge that the signal detection models' being a class of the latent variable models could also be formulated within the structural equation modeling framework (Bollen, 1989) with the estimation procedure accomplished by LISREL (Jöreskog \& Sörbom, 1993). The regression approach is, however, likely to be more familiar to psychologists and is readily applicable to experimental data. Furthermore, the nonlinear regression methodology allows reasonable non-normal models such as logistic or complementary $\log -\log$ distributions to be implemented with minimal changes in program code.

In this article, we first briefly review the signal detection model for rating data. The second section illustrates Tosteson and Begg's (1988) approach by formulating a regression model for Kijewski et al.'s (1989) data from a multiple-alternative perceptual task. The third section describes the details of using the SAS nonlinear regression program for nonlinear model fitting. The last section presents an application of the regression approach to confidence rating data from a recognition memory experiment.

\section{The Signal Detection Model for Rating-ROC Data}

This section illustrates the construction of signal detection models for rating data and introduces notation. Subjects in a discrimination experiment are asked to use a fixed number of categories to indicate how confident they are that a signal has been presented in an experimental trial. For example, four response categories from $1=$ noise for sure to $4=$ signal for sure may be used. A normal model for this case assumes a normal distribution for noise, with mean $\mu_{1}$ and standard deviation $\sigma_{1}$, and a normal distribution for signal, with mean $\mu_{2}$ and standard deviation $\sigma_{2}$. To fit four categorical responses, three (latent) cutpoints are assumed. Thus, depending on whether a noise or a signal stimulus has been presented, we have ( $\Phi$ denotes the cumulative distribution function of a standard normal random variable):

$$
\begin{aligned}
& \operatorname{Pr}\left(R=1 \mid S_{i}\right)=\Phi\left(\left(c_{1}-\mu_{i}\right) / \sigma_{i}\right), \\
& \operatorname{Pr}\left(R=2 \mid S_{i}\right)=\Phi\left(\left(c_{2}-\mu_{i}\right) / \sigma_{i}\right)-\Phi\left(\left(c_{1}-\mu_{i}\right) / \sigma_{i}\right), \\
& \operatorname{Pr}\left(R=3 \mid S_{i}\right)=\Phi\left(\left(c_{3}-\mu_{i}\right) / \sigma_{i}\right)-\Phi\left(\left(c_{2}-\mu_{i}\right) / \sigma_{i}\right), \\
& \operatorname{Pr}\left(R=4 \mid S_{i}\right)=1-\Phi\left(\left(c_{3}-\mu_{i}\right) / \sigma_{i}\right),
\end{aligned}
$$

where $R$ is the categorical response for a particular trial in the experiment, and $c_{1}, c_{2}$, and $c_{3}$ are the cutpoints (decision thresholds). A subject's categorical responses are used to estimate the location-shift parameter $\left(\mu_{2}-\mu_{1}\right)$, the scale parameter $\left(\sigma_{1} / \sigma_{2}\right)$, and the cutpoints. Typically, $\mu_{1}$ is set to 0 and $\sigma_{1}$ to 1 for identifiability. This is equivalent to choosing the noise distribution as the reference for comparison. Note that, if the noise and signal distributions have the same variance, the scale parameter reduces to a constant of 1 . This is the equal-variance normal model, and it belongs to the class of generalized linear models. In the case where noise and signal distributions have different variances, the constraint that the scale parameter has to be positive excludes the unequalvariance normal model from the family of the generalized linear models. Hence, the unequal-variance model must be estimated with the use of the maximum-likelihoodmethod presented in Dorfman and Alf (1969) or a nonlinear regression procedure such as that proposed by Cox (1984).

\section{The Regression Approach to ROC Data}

Tosteson and Begg (1988) proposed a generalization of the signal detection models, allowing the shift in location and/or scaling to depend on each of the distributions (noise or signal) under consideration. This family of models also appeared in McCullagh and Nelder (1989, p. 154). We present a regression model of this type with a single continuous covariate for data from a perceptual experiment reported in Kijewski et al. (1989). One observer's 12-category rating of 196 visual stimuli were recorded under five levels of stimulus contrast: $-2,-1,0$, 1 , and 2. The number of stimulus presentations (trials) for each stimulus contrast was $38,38,40,40$, and 38 , respectively. The model proposed by Kijewski et al.'s has a total of 19 parameters: a pair of location and scale parameters for each of the four distributions corresponding to 
non-zero contrast levels plus 11 cutpoint parameters. We noticed that the parameter estimates reported in Kijewski et al.'s paper were linearly related to the stimulus contrast levels, so we propose a simple model with one location parameter and one scale parameter that determines the mean and standard deviation of each condition as a linear function of contrast.

Let $\pi_{i j}$ be the probability of $j$ th categorical response $(j=1,2, \cdots 12)$ for distribution $i(i=1, \cdots 5)$. For example, $\pi_{36}$ is the (theoretical) probability of responding with a judgment of category 6 when the level of stimulus contrast is 0 . The observed frequency of this categorical response was 14 . These multinomial probabilities can be determined by a normal model for 12 categories and 5 distributions, similar to the normal model described earlier for 4 categories and 2 distributions. We will assume that the distribution for the 0 -contrast $(i=3)$ condition is used as reference with a mean of 0 and variance of one. The model we consider has a total of 13 parameters and takes the following form:

$$
p_{i j}=\Phi\left(\frac{c_{j}-\beta x_{i}}{\exp \left(\alpha x_{i}\right)}\right)
$$

where $p_{i j}=\pi_{1 j}+\cdots+\pi_{i j}$ is the cumulative probability for each of the 12 categories at the stimulus contrast level $x_{i}$, with $x_{1}=-2, x_{2}=-1, x_{3}=0, x_{4}=1$, and $x_{5}=$ $2 ; c_{1} \cdots c_{11}$ are the cutpoints for the 12 categorical responses. The scale parameter is exponentiated to ensure non-negative variance estimates. It can be shown that $\exp (-\alpha)$ is the ratio of standard deviations between the reference distribution ( 0 contrast) and any of the distributions corresponding to the non-zero stimulus contrast.

\section{Parameter Estimation by Nonlinear Regression}

At present, fitting procedures for models such as those specified by Equation 1 do not exist in general purpose statistical software, although some procedures are available for specific cases (Kang Fu, 1998). Typically, specialized computer programs have been used. Tosteson and Begg (1988) used PLUM, an interactive package written in FORTRAN, for their analysis. On the other hand, standard nonlinear regression programs are available in BMDP, SAS, and SPLUS. The approach proposed by Cox (1984) was to treat the problem of maximizing the likelihood as a regression problem (Jennrich \& Moore, 1975). This approach requires a nonlinear regression program that uses the Gauss-Newton algorithm in iteratively reweighted mode. The program used in the present paper is SAS PROC NLIN (SAS Institute, 1999). Our presentation assumes only minimal knowledge of SAS, and listings are provided for the first example. The price and availability of this statistical package can be obtained at www.sas.com.

To fit rating data by ordinal regression using PROC NLIN, the user specifies the predicted probabilities for each categorical response as a function of the parameters using Equation 1 and the weights, which are the inverses of the predicted frequencies multiplied by the sample size of the particular condition. The weights correspond to elements of a generalized inverse of the multinomial covariance matrix (see McCullagh \& Nelder, 1989, p. 168). PROC NLIN iteratively updates the weights while the parameter estimates (and hence the predicted frequencies) are updated. By default, the residual sum of squares is used by PROC NLIN as the quantity to be minimized. For our purpose, the default loss function (LOSS is a SAS keyword) is replaced by the deviance. The deviance for the multinomial distribution is

$$
G^{2}=-2 \sum_{i, j} y_{i j} \ln \left(\hat{\pi}_{i j} / p_{i j}\right)
$$

where $y_{i j}$ is the observed count in category $j$ of condition $i$ and $\pi_{i j}$ is the fitted probability of $j$ th categorical response for distribution $i$. Differences between values of deviance at convergence can be used for comparing the goodness-of-fit of nested models. For large samples, the difference in $G^{2}$ is approximately distributed as $\chi^{2}$ with degrees of freedoms equal to the number of parameters of the full model minus the number of parameters of the reduced model. The Pearson $\chi^{2}$ statistic is a quadratic approximation for the $G^{2}$ statistic (Agresti, 1996). The higher-order terms in the $G^{2}$ statistic become negligible only when the model holds and the sample size is sufficiently large. The $G^{2}$ statistic is typically adopted for model comparison in the generalized linear model framework.

The user can also specify the partial derivatives with respect to the model parameters or choose the derivativefree algorithm (which computes the required derivatives numerically) with the METHOD=DUD option. Other options in PROC NLIN allow the user to specify bounds on the values of the parameters, set a grid of possible starting values and a criterion of convergence and a method of selecting the step-size used by the optimizationalgorithm.

The next section presents the results of fitting Equation 1 to the rating data reported in Kijewski et al. (1989). The nonlinear regression approach has two advantages: (1) The implementation does not require derivations of second-order derivatives. (2) The parameters specified in Equation 1 are regression coefficients and, hence, can be directly evaluated. For instance, if the estimated value of the scale parameter $\alpha$ in our 13-parameter model is close to 0 this suggests that an equal variance model can account for Kijewski et al.'s data.

\section{Analysis of Rating Data From Multiple-Alternative Tasks}

Listing 1 presents the SAS code for fitting Equation 1 to multiple-alternative rating data with a continuous covariate representing the level of stimulus contrasts. The top and bottom seven lines in the input (data) file to the program are shown in Listing 2. The parameter estimates and the goodness-of-fit index (deviance) are shown in Listing 3. The value next to Objective in the output is the minimum deviance.

Inspection of Listing 3 suggests an equal variance model applies, because the estimate of the scale parameter, $\alpha$ (a in the SAS listing), is close to 0 . This suggests a 12- 
Listing 1

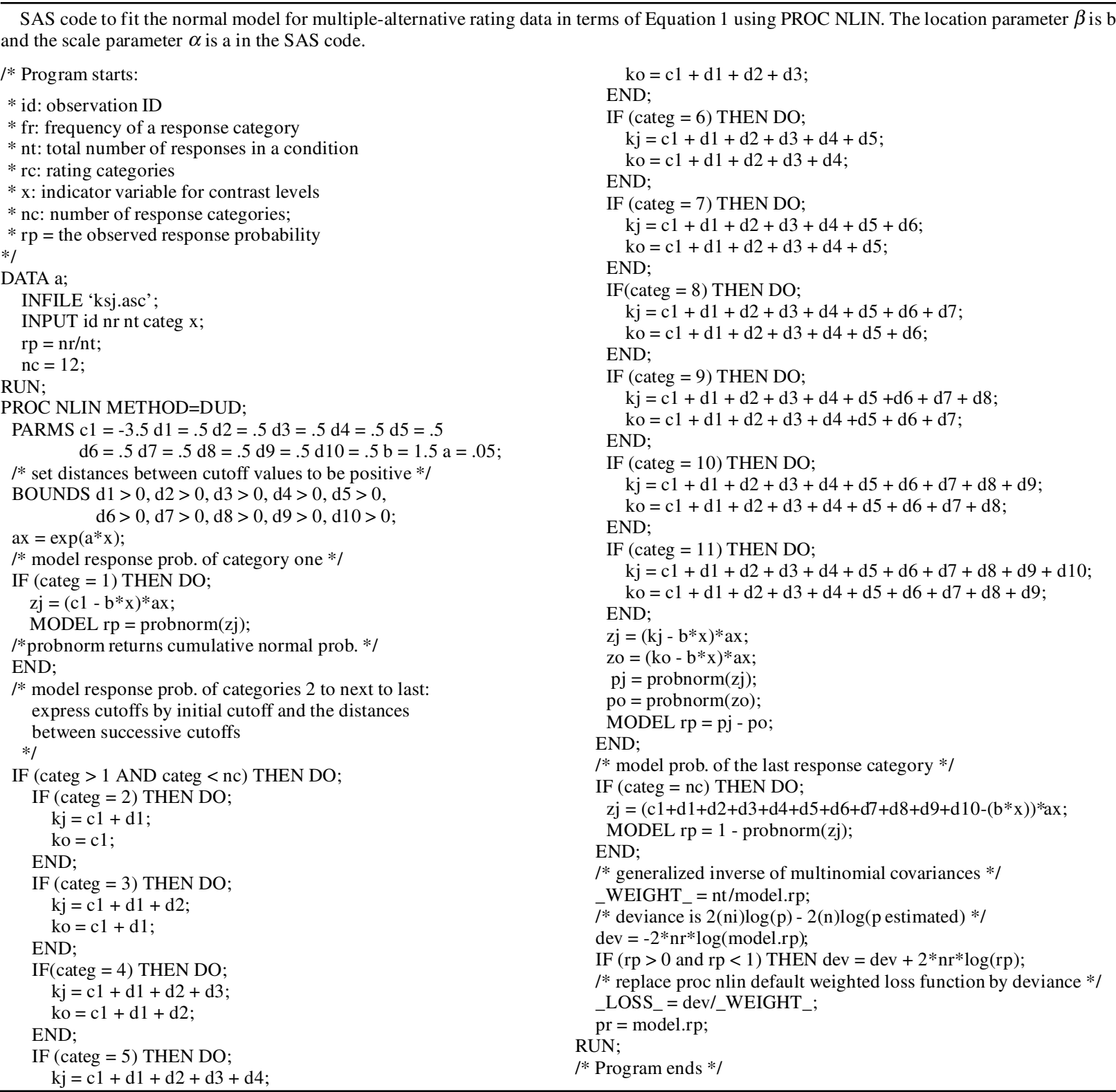

parameter model with one location parameter and 11 cutpoint parameters. This model corresponds to the equalvariance case of a normal model and is a member of the generalized linear model family. Thus, SAS PROC LOGISTIC with a probit link can also be used to fit the simpler 12-parameter model directly. The unequal-variance model as specified in Equation 1, on the other hand, is not a generalized linear model, because it is nonlinear in the parameters. It should be pointed out that nonlinearity in parameters does not imply a nonlinear relationship between the $z$-transformed hits and false alarms. What it does imply is that the slope parameter for relating $z$-transformed false alarms to $z$-transformed hits is not equal to unity. The equal- and unequal-variance models are, however, nested, and the difference between values of the $G^{2}$ statistic (deviance) can be used to test whether the fits between the full and reduced models are significantly different. The results of the equal-variance model fit using SAS PROC NLIN are shown in Listing 4. The deviance measures (Objective) are almost identical for both the full model and the reduced model, indicating that there is no evidence for unequal variance. The $G^{2}$ value of 36.91 with 43 degrees of freedom indicates that the equal-variance normal models using the level of stimu- 
Listing 2

The first and last five lines of data file to SAS program of Listing 1. The contrast level is treated as a quantitative variable. Column 1: observation ID; Column 2: number of responses; Column 3: total number of responses in a condition; Column 4: response category; and Column 5: stimulus contrast level.

\begin{tabular}{rrrrr}
1 & 19 & 38 & 1 & -2 \\
2 & 14 & 38 & 2 & -2 \\
3 & 3 & 38 & 3 & -2 \\
4 & 2 & 38 & 4 & -2 \\
5 & 0 & 38 & 5 & -2 \\
.. &. & $\ldots$ & $\ldots$ & $\ldots$ \\
56 & 1 & 38 & 8 & 2 \\
57 & 4 & 38 & 9 & 2 \\
58 & 12 & 38 & 10 & 2 \\
59 & 8 & 38 & 11 & 2 \\
60 & 13 & 38 & 12 & 2 \\
\hline
\end{tabular}

lus contrast as a quantitative covariate fits as well as $\mathrm{Ki}$ jewski et al.'s 19-parameter model. They reported a $\chi^{2}$ value of 31.61 with 36 degrees of freedom.

Listing 5 displays the results of fitting the equal-variance normal model using SAS PROC LOGISTIC. As expected, the results are the same as those obtained by the nonlinear regression procedure, demonstrating that maximumlikelihood estimates of signal detection parameters can be obtained by a nonlinear regression procedure, as discussed in the previous section. The intercept parameter estimates in the PROC LOGISTIC are the cutpoint estimates; the slope estimate (b in Listing 5) corresponds to the location-shift caused by a unit increase in the stimulus contrast level.

\section{Application:}

\section{Frequency Effect in Recognition Memory}

McAuley and Heathcote (1999) conducted an experiment on recognition memory for words using a studytest paradigm. They used a within-subjects design with three levels of word frequency (low, medium, and high) crossed with two levels of new (unstudied) versus old (studied) words. Thus, there were six treatment combinations. The subjects were asked to provide ratings from 1 to 6 to indicate their confidence that a word was new $(1=$ sure new to $3=$ possibly new $)$ or old $(4=$ possibly old to $6=$ sure old $)$. It was expected that the subjects would be able to discriminate between new and old words and that discriminability would be highest for the condition in which low-frequency words were used and lowest for the condition in which high-frequency words were used. To use the regression approach, we constructed a design matrix for the $2 \times 3$ factorial design. We used one indicator variable to code the new-old word condition $($ old $=1$, new $=0$ ) and two indicator variables for the three levels of word-frequency factor. For the wordfrequency factor, we used reference cell coding indicating membership of the medium- and low-frequency conditions, respectively. The new high-frequency word condition served as the reference group (intercept) in this regression model. Listing 6 shows the top 10 rows of the input file for one subject in the experiment.

Let $p_{i, j}$ be the probability of making a $j$ th categorical response in the $i$ th condition. A condition is defined by three indicator variables, $x_{i, 1}, x_{i, 2}$, and $x_{i, 3}$, the first for the new-old word condition and the latter two for mediumand low-frequency words, respectively. We model the cumulative probability $\pi_{i, j}=p_{i, 1}+\cdots p_{i, j}(1 \leq j \leq k-1$, $k=6$ ) by

$$
\pi_{i, j}=\Phi\left(\frac{c_{1}+d_{1}+\cdots+d_{j-1}-\left(\beta_{1} x_{i, 1}+\beta_{2} x_{i, 2}+\beta_{3} x_{i, 3}\right)}{\exp \left(\alpha x_{i, 1}\right)}\right),
$$

where $c_{1}$ is the first cut point, and $d_{l}=c_{l+1}-c_{l}, l=1$, $\cdots 4$. The distribution for latent responses evoked by the presentation of stimulus in the new high-frequency word condition assumes a standard normal distribution. In this

Listing 3

Edited SAS output of PROC NLIN for unequal-variance normal models with the level of contrasts treated as a quantitative variable. The objective function to be minimized is the deviance.

$$
\begin{aligned}
& \text { The NLIN Procedure } \\
& \text { Estimation Summary } \\
& \text { Objective } 36.90741
\end{aligned}
$$

\begin{tabular}{ccccc} 
Parameter & Estimate & $\begin{array}{c}\text { Approx } \\
\text { Std Error }\end{array}$ & Approximate & 95\% Confidence Limits \\
c1 & -3.3685 & 0.2552 & -3.8819 & -2.8551 \\
d1 & 0.8743 & 0.1928 & 0.4865 & 1.2620 \\
d2 & 0.7006 & 0.1649 & 0.3689 & 1.0324 \\
d3 & 0.5223 & 0.1438 & 0.2330 & 0.8115 \\
d4 & 1.2979 & 0.2099 & 0.8756 & 1.7202 \\
d5 & 0.5685 & 0.1465 & 0.2739 & 0.8632 \\
d6 & 0.6116 & 0.1511 & 0.3077 & 0.9154 \\
d7 & 0.5495 & 0.1445 & 0.2588 & 0.8402 \\
d8 & 0.6723 & 0.1595 & 0.3515 & 0.9932 \\
d9 & 1.0107 & 0.2044 & 0.5996 & 1.4219 \\
d10 & 0.4888 & 0.1681 & 0.1507 & 0.8269 \\
b & 1.7070 & 0.1151 & 1.4755 & 1.9386 \\
a & -0.00078 & 0.0632 & -0.1279 & 0.1263 \\
\hline
\end{tabular}


Listing 4

Edited SAS output of PROC NLIN for the equal-variance normal models. The model assumes the same scale parameter for the distributions of all conditions.

\begin{tabular}{|c|c|c|c|c|}
\hline \multicolumn{5}{|c|}{ The NLIN Procedure } \\
\hline & Method & & & DUD \\
\hline & Iterations & & & 93 \\
\hline & Objective & & & 36.90754 \\
\hline & & Approx & & \\
\hline Parameter & Estimate & Std Error & Approximate & 95\% Confidence Limits \\
\hline c1 & -3.3680 & 0.2495 & -3.8695 & -2.8664 \\
\hline $\mathrm{d} 1$ & 0.8734 & 0.1814 & 0.5086 & 1.2381 \\
\hline $\mathrm{d} 2$ & 0.7004 & 0.1612 & 0.3763 & 1.0245 \\
\hline d3 & 0.5221 & 0.1417 & 0.2372 & 0.8071 \\
\hline $\mathrm{d} 4$ & 1.2980 & 0.2076 & 0.8806 & 1.7154 \\
\hline d5 & 0.5686 & 0.1448 & 0.2774 & 0.8597 \\
\hline d6 & 0.6116 & 0.1493 & 0.3114 & 0.9118 \\
\hline d7 & 0.5496 & 0.1424 & 0.2632 & 0.8360 \\
\hline $\mathrm{d} 8$ & 0.6728 & 0.1559 & 0.3593 & 0.9863 \\
\hline d9 & 1.0115 & 0.1910 & 0.6275 & 1.3955 \\
\hline $\mathrm{d} 10$ & 0.4896 & 0.1598 & 0.1683 & 0.8108 \\
\hline $\mathrm{b}$ & 1.7070 & 0.1137 & 1.4783 & 1.9357 \\
\hline
\end{tabular}

Listing 5

\begin{tabular}{|c|c|c|c|c|c|}
\hline \multicolumn{6}{|c|}{ Edited SAS output of equal-variance normal model using SAS PROC LOGISTIC. } \\
\hline \multicolumn{6}{|c|}{$\begin{array}{l}\text { The LOGISTIC Procedure } \\
\text { Deviance and Pearson Goodness-of-Fit Statistics }\end{array}$} \\
\hline Criterion & & DF & Value & Value/DF & $\operatorname{Pr}>$ Chi-Square \\
\hline Deviance & & 43 & 36.9075 & 0.8583 & 0.7317 \\
\hline Pearson & & 43 & 49.9615 & 1.1619 & 0.2163 \\
\hline \multicolumn{6}{|c|}{ Analysis of Maximum Likelihood Estimates } \\
\hline & & Parameter & r Standard & Wald & \\
\hline Variable & DF & Estimate & Error & Chi-Square & $\operatorname{Pr}>$ Chi-Square \\
\hline $\mathrm{C} 1$ & 1 & -3.3680 & 0.2446 & 189.6588 & 0.0001 \\
\hline $\mathrm{C} 2$ & 1 & -2.4946 & 0.2106 & 140.2986 & 0.0001 \\
\hline $\mathrm{C} 3$ & 1 & -1.7943 & 0.1859 & 93.1675 & 0.0001 \\
\hline $\mathrm{C} 4$ & 1 & -1.2721 & 0.1710 & 55.3388 & 0.0001 \\
\hline $\mathrm{C} 5$ & 1 & 0.0259 & 0.1493 & 0.0300 & 0.8625 \\
\hline C6 & 1 & 0.5944 & 0.1531 & 15.0705 & 0.0001 \\
\hline C7 & 1 & 1.2060 & 0.1656 & 53.0559 & 0.0001 \\
\hline $\mathrm{C} 8$ & 1 & 1.7557 & 0.1816 & 93.4516 & 0.0001 \\
\hline C9 & 1 & 2.4285 & 0.2055 & 139.6923 & 0.0001 \\
\hline $\mathrm{C} 10$ & 1 & 3.4400 & 0.2467 & 194.4439 & 0.0001 \\
\hline $\mathrm{C} 11$ & 1 & 3.9295 & 0.2695 & 212.5574 & 0.0001 \\
\hline $\mathrm{b}$ & 1 & 1.7070 & 0.1115 & 234.2915 & 0.0001 \\
\hline
\end{tabular}

formulation, the ratio of standard deviations between the reference distribution and any of the signal conditions is represented by $\exp (-\alpha)$. If $\alpha$ is not equal to 0 , we have an unequal-variance model (Ratcliff, Sheu, \& Gronlund, 1992). Preliminary model fitting achieved satisfactory goodness-of-fit of the model holding the value of the $\alpha$ parameter at a non-zero constant. This means that all the signal conditions relative to the reference condition have the same variance ratio. This is a much simpler model than one with a different scale parameter for each of the conditions relative to the reference condition. The parameter $\beta_{1}$ represents the location shift from the reference distribution to that of the old high-frequency word condition; the sum $\beta_{1}+\beta_{2}$ represents the location shift from the reference distribution to the of old medium-frequency word condition; and $\beta_{1}+\beta_{3}$ represents the location shift from the reference distribution to the of old low-frequency word condition.

To illustrate the flexibility of the regression approach, we tested the hypothesis that parameter location increases as word frequency decreases. To do so, we estimated the $\beta$ parameters under both the restricted-order constraint $\left(\beta_{1} \geq 0 ; \beta_{3} \geq \beta_{2} \geq 0\right)$ and the unrestricted condition $\left(\beta_{1} \geq 0\right.$; $\beta_{2} \geq 0 ; \beta_{3} \geq 0$ ). To constrain order, we use a difference parameterization and bounding below by zero (the BOUNDS statement in SAS restrains the parameter estimates within specific bounds. See, for example, Listing 1). Note that the parameter estimates cannot be constrained with the use of the traditional fitting procedures of ROC data. Hence, one must conduct a test of homogeneity of means 


\section{Listing 6}

The first 10 lines of data file from a participant in the word recognition experiment (McAuley \& Heathcote, 1999). Column 1: subject ID; Column 2: response frequency in each of six response categories; Column 3: total response frequencies for a condition; Column 4: response categories; Column 5: word-frequency condition, 1 = high, 2 = medium, 3 = low; Column 6: new-old word condition, new $=0$, old $=1$; Column 7: medium-frequency indicator variable, high $=0$, medium $=1$, and low $=0$; Column 8 : low-frequency indicator variable, high $=0$, medium $=0$, low $=1$

\begin{tabular}{lrllllll}
$\mathrm{S} 1$ & 69 & 150 & 1 & 1 & 0 & 0 & 0 \\
$\mathrm{~S} 1$ & 41 & 150 & 2 & 1 & 0 & 0 & 0 \\
$\mathrm{~S} 1$ & 19 & 150 & 3 & 1 & 0 & 0 & 0 \\
$\mathrm{~S} 1$ & 16 & 150 & 4 & 1 & 0 & 0 & 0 \\
$\mathrm{~S} 1$ & 4 & 150 & 5 & 1 & 0 & 0 & 0 \\
$\mathrm{~S} 1$ & 1 & 150 & 6 & 1 & 0 & 0 & 0 \\
$\mathrm{~S} 1$ & 46 & 150 & 1 & 2 & 0 & 1 & 0 \\
$\mathrm{~S} 1$ & 52 & 150 & 2 & 2 & 0 & 1 & 0 \\
$\mathrm{~S} 1$ & 30 & 150 & 3 & 2 & 0 & 1 & 0 \\
$\mathrm{~S} 1$ & 14 & 150 & 4 & 2 & 0 & 1 & 0 \\
\hline
\end{tabular}

among the "population" of $\beta$ parameters under restrictive alternatives, such as those given above (Bartholomew, 1959), after extracting the parameter estimates. Unfortunately, the computation for such a test procedure is quite involved and is not available in standard statistical software packages. Because difference parameterization and zero bounding constraints are used with our methods, in contrast, the parameters can be estimated easily.

Our results show that the model fits data for 27 out of 32 subjects (not rejected at $\beta=.05$ level of significance) when the $\beta$ parameters were not restricted. Inspection of the standardized residual plots reveals that the model fits were inadequate for only 4 subjects. The model estimation under order constraint fit 23 out of 32 subjects (with the same 23 subjects among the 27 subjects in the unrestricted case). The parameter estimates are very similar for all but 3 of the 4 subjects where only the order-unrestricted model fit well. The average parameter estimates of 23 subjects (under order constraint) are $\beta_{1}=1.874, \beta_{2}=$ .137 , and $\beta_{3}=.326$ for the location shifts and the ratio of standard deviations, $\exp (-\alpha)=.717$. We interpret this as direct evidence in support of the word-frequency effect in a recognition memory experiment and conclude that there is evidence for a word-frequency effect on location, with location being ordered by word frequency for the majority of subjects.

\section{CONCLUSION}

Signal detection models play an important role in the analysis of rating data from cognitive, perceptual, and memory experiments. The ordinal regression modeling methods proposed by Tosteson and Begg (1988) allow the experimenter to assess the effects of treatment factors directly by examining regression coefficients, and they allow for the possibility of both covariates to be adjusted to location and scale parameters. Cox (1984) showed that the extensions of generalized linear models such as the general model proposed by Tosteson and Begg can be fitted by iteratively reweighted least squares. The use of SAS PROC NLIN provides a convenient and flexible platform for model fitting. The user can place bounds on the parameters as well as conduct an initial grid search. The derivative-free algorithm can be used when the derivatives are difficult to specify or when the derivatives are obtained from software that does symbolic computation. The advantage of the regression approach is that one does not need to devote a large amount of effort to modifying a specialized computer program in order to compare a variety of models, as is commonly required for nested model testing.

\section{REFERENCES}

Agresti, A. (1996). An introduction to categorical data analysis. New York: Wiley.

Bartholomew, D. J. (1959). A test of homogeneity for ordered alternatives. Biometrika, 46, 36-48.

Bollen, K. A. (1989). Structural equations with latent variables. New York: Wiley.

Cox, C. (1984). Generalized linear models-the missing link. Applied Statistics, 33, 18-24.

DeCARLO, L. T. (1998). Signal detection theory and generalized linear models. Psychological Methods, 3, 186-205.

Dorfman, D. D., \& Alf, E. (1969). Maximum-likelihood estimation of parameters of signal detection theory and determination of confidence intervals - rating-method data. Journal of Mathematical Psychology, 6, 487-496.

Green, D. M., \& Swets, J. A. (1974). Signal detection theory and psychophysics. Huntington, New York: Krieger.

JENNRICH, R. I., \& Moore, R. H. (1975). Maximum likelihood estimation by means of nonlinear least squares. Proceedings of the Statistical Computing Section of the American Statistical Association, pp. 57 65.

Jöreskog, K. G., \& Sörbom, D. (1993). LISREL 8.14. Chicago: Scientific Software International.

KANG Fu, V. (1998). Estimating generalized ordered logit models. Stata Technical Bulletin, 44, 27-30.

KiJewski, M. F., Swensson, R. G., \& Judy, P. F. (1989). Analysis of rating data from multiple-alternative tasks. Journal of Mathematical Psychology, 33, 423-451.

MacMillan, N. A., \& Creelman, C. D. (1991). Detection theory: A user's guide. New York: Cambridge University Press.

McAuley, M., \& Heathcote, A. (1999). Psycholinguistic properties and the mirror effect: More than one cause? Poster session presented at the Australian Experimental Psychology Conference, Sydney.

McCullagh, P., \& Nelder, J. A. (1989). Generalized linear models. New York: Chapman \& Hall.

Ratcliff, R, McKoon, G., \& Tindall, M. (1994). Empirical generality of data from recognition memory receiver-operating characteristic functions and implication for the global memory models. Journal of Experimental Psychology: Learning, Memory, \& Cognition, 20, 763-785.

Ratcliff, R., Sheu, C.-F., \& Gronlund, S. D. (1992). Testing global memory models using ROC curves. Psychological Review, 99, 518-535.

SAS Institute, Inc. (1999). SAS/STAT User's Guide (Version 8). Cary, NC: Author.

Tosteson, A. A., \& Begg, C. B. (1988). A general regression methodology for ROC curve estimation. Medical Decision Making, 8, 204-215.

(Manuscript received November 24, 2000; revision accepted for publication March 13, 2001.) 